\author{
Revue \\ de /'histoire \\ Revue de l'histoire des religions \\ des religions \\ $4 \mid 2014$ \\ L'objet rituel. Concepts et méthodes croisés
}

\title{
L'objet et son image dans le rituel
}

Contribution à la connaissance des usages cultuels en Géorgie au début du Moyen Âge

The object and its image in the ritual. Exploring cultic practices in Georgia in the early Middle Ages

Nina lamanidzé

\section{OpenEdition}

Journals

Édition électronique

URL : http://journals.openedition.org/rhr/8335

DOI : 10.4000/rhr.8335

ISSN : 2105-2573

Éditeur

Armand Colin

Édition imprimée

Date de publication : 1 décembre 2014

Pagination : 797-817

ISSN : 0035-1423

Référence électronique

Nina lamanidzé, «L'objet et son image dans le rituel », Revue de l'histoire des religions [En ligne],

4 | 2014, mis en ligne le 01 décembre 2017, consulté le 01 mai 2019. URL : http://

journals.openedition.org/rhr/8335; DOI : 10.4000/rhr.8335 


\section{L'objet et son image dans le rituel Contribution à la connaissance des usages cultuels en Géorgie au début du Moyen Âge}

L'étude tente d'établir les rapports entre l'objet, l'image sur l'objet et la liturgie proprement dite à travers le matériel archéologique géorgien, qui propose un usage des images où la richesse et la complexité des programmes vont au-delà de la simple définition iconographique. Le recours fréquent aux représentations d'objets rituels révèle que le répertoire des monuments géorgiens au début du Moyen Âge puise non seulement dans les textes bibliques et hagiographiques mais aussi dans le rituel, notamment dans la divine liturgie. Cette référence à la liturgie permet d'établir la fonction de l'objet et, dans certains cas, d'émettre des hypothèses sur la reconstitution du rituel.

The object and its image in the ritual.

Exploring cultic practices in Georgia in the early Middle Ages

This study tries to establish the relationship between the object, the image on the object and the liturgy through the Georgian archaeological evidence, which offers a use of images in which the richness and complexity of the programs go beyond a simple iconographic definition. The frequent use of the representation of ritual objects reveals that the repertoire of Georgian monuments of the early Middle Ages draws not only on biblical and hagiographic texts but also on the ritual, especially on the Divine Liturgy. This reference to the liturgy establishes the function of the object and, in some cases, enables us to make assumptions about the reconstitution of the ritual itself. 
Christianisée au début de $\mathrm{IV}^{\mathrm{e}}$ siècle, la Géorgie a connu un long cheminement de développement culturel. Cette composante majeure de l'Orient chrétien, qui a assimilé les rites et les traditions de la religion chrétienne, a activement contribué au processus de développement de l'art de l'Orient byzantin. Le corpus géorgien des images en usage au cours des $\mathrm{VI}^{\mathrm{e}}-\mathrm{X}^{\mathrm{e}}$ siècles rassemble un vaste groupe de monuments et d'objets cultuels, remarquables par leur décor et leur précocité. La sculpture apparaît comme l'expression artistique la plus caractéristique de cette époque, quand les concepteurs géorgiens recouraient rarement aux autres moyens d'expression artistique. Ce matériel archéologique demeure mal connu et pose de nombreux problèmes d'interprétation; l'une des principales difficultés, particulièrement épineuse, concerne l'établissement des rapports entre l'objet, l'image sur l'objet et la liturgie proprement dite. La première précaution pour l'aborder est de ne pas englober sous le terme de rituel ce qui relève plutôt d'usages dévotionnels, privilégiés par certains donateurs. Il s'agit aussi d'éviter de surinterpréter ce qui procède, dans l'iconographie, d'une simple illustration des fondements du dogme général du christianisme, illustration qui se limite à articuler les croyances chrétiennes générales. Il faut donc distinguer ces dernières de ce qui relève de la relation entre croyances et actes qui sont reproduits pendant le service, conduits autour des objets. La pauvreté de sources écrites appropriées ne facilite pas cette tâche. Il n'existe en effet que de très rares témoignages textuels sur le déroulement de la liturgie dans l'Église orthodoxe géorgienne avant le $\mathrm{X}^{\mathrm{e}}$ siècle. Les origines et les buts des pratiques rituelles restent obscurs, ce qui rend ardue la reconstitution des fonctions liturgiques de certaines œuvres et les usages que leur réservaient les fidèles. On se posera alors les questions de savoir comment l'image sur l'objet peut aider à reconstituer la fonction cultuelle et comment l'image de l'objet même aide à identifier le rituel.

\section{LES STÈLES EN PIERRE : CONTEXTES ET DÉCORS}

Les stèles sculptées géorgiennes constituent un corpus remarquablement riche qui, malgré sa grande diffusion en Géorgie, dès la 
fin du $\mathrm{IV}^{\mathrm{e}}$ siècle et particulièrement aux $\mathrm{VI}^{\mathrm{e}}$ et $\mathrm{VII}^{\mathrm{e}}$ siècles, demeure peu connu des non spécialistes. Ces croix sculptées érigées sur les piliers richement ornés de reliefs ont été installées en plein air, dans des espaces ruraux, souvent assez loin des églises. Les représentations figuratives commencent à apparaître, se multiplier et s'agréger au sein de programmes complexes sur les stèles à partir de la deuxième moitié $\mathrm{du} \mathrm{VI}^{\mathrm{e}}$ siècle, ce qui correspond à la date où le culte des images chrétiennes augmente et s'intensifie en général'. Le grand nombre de ces objets monumentaux conservés confirme qu'à l'époque paléochrétienne il existait en Géorgie un système bien organisé de production de stèles et que la répartition géographique des ateliers était très large dans les différentes régions du pays.

Les sources historiques offrent des renseignements sur les premières stèles géorgiennes: les chroniques de Mok'cevay K'art'lisay (la conversion de K'art'li) de Leonti Mroveli rapportent l'histoire de la construction d'une croix en bois à Mcxet'a par le roi de K'art'li Mirian au début du IV $\mathrm{IV}^{\mathrm{e}}$ siècle, après son baptême ${ }^{2}$. La croix de Mcxet'a est ainsi devenue le symbole du christianisme et du baptême. La même source - Mok'cevay K'art'lisay - affirme que sainte Nino, l'illuminatrice de la Géorgie, a ordonné de détruire les anciennes idoles et a érigé à leur place des stèles, symboles de la nouvelle religion. Les chroniques de K'art'lis cxovreba (la vie de K'art'li) confirment que les croix en bois installées aux mêmes endroits que les anciennes idoles païennes ont été progressivement remplacées par des stèles en pierre et se sont répandues dans toute la Géorgie ${ }^{3}$. Les récits des pèlerins attestent le fait que l'installation de stèles surmontées d'une croix est liée à la reconnaissance du christianisme, confirmée par l'acte du baptême. Les pèlerins, voyageant à Jérusalem dans les premières années du christianisme, ont certainement vu la croix installée dans le Jourdain, à l'emplacement du baptême du Christ ${ }^{4}$. Cependant ces textes ne fournissent

1. André Grabar, Martyrium. Recherches sur le culte des reliques et l'art chrétien antique, Paris, 1946, p. 343; Ernst Kitzinger, «The Cult of Images in the Age before Iconoclasm», Dumbarton Oaks Papers 9, 1954, p. 95-97.

2. K'art'lis cxovreba (La vie de K'art'li), Simon Q'auxčišvili éd., Tbilissi, 1955, vol. I, p. 72-130.

3. K'art'lis cxovreba, vol. I, p. 72-130.

4. Anatole Frolow, «Numismatique byzantine et archéologie des lieux saints. Au sujet d'une monnaie de l'impératrice Eudocie ( $v^{\mathrm{e}}$ siècle)», Mémorial Luis Petit. Mélanges d'histoire et d'archéologie byzantines («Archives de l'Orient 
pas de renseignements précis sur la fonction de ces objets. L'image reste ainsi la seule source permettant de déterminer la fonction de ces stèles.

Le relief de la façade est de l'église d'Edzani, du $\mathrm{VI}^{\mathrm{e}} \mathrm{s}$., reproduit entièrement la structure de ces stèles et leur typologie: ce sont des piliers, le plus souvent carrés, habituellement ornés de reliefs, montés sur une base cubique et surmontés d'une croix.

L'image du chapiteau de la stèle de Dmanisi ( $\mathrm{vi}^{\mathrm{e}} \mathrm{s}$.) fournit davantage de renseignements encore. Les deux faces de ce fragment cubique offrent une représentation identique: montées sur des bases ornementées, des croix de Malte, très fréquentes en Géorgie, surtout au $\mathrm{VI}^{\mathrm{e}}$ siècle $^{5}$, portent au croisement des bras un médaillon imitant une pierre précieuse; deux autres médaillons analogues sont suspendus aux bras horizontaux de la croix. Une troisième face présente une image semblable mais la croix est remplacée par une rosette ou plutôt par une variante très stylisée de chrisme, étroitement lié à la croix qu'il remplace ici'. Une quatrième face (fig. 1) montre une stèle décorative couronnée d'une croix, avec à ses côtés un personnage assis sous une arche, les jambes croisées à l'orientale.

Le décor du chapiteau de Dmanisi exalte et glorifie la croix : répétée plusieurs fois sur cet objet, décorée de pierres précieuses, elle renvoie à la croix dressée sur le Golgotha dès l'époque constantinienne, qui fut remplacée par Théodose II par une croix décorée de pierres précieuses. La stèle reproduit le type traditionnellement employé à l'époque paléochrétienne pour évoquer la croix de Jérusalem, décrite par les pèlerins et figurée sur les ampoules et sur d'autres objets liturgiques paléochrétiens ${ }^{7}$. Ainsi cette image atteste la tradition de la vénération de la croix en Géorgie paléochrétienne.

chrétien», 1), Paris, 1948, p. 83-85, avec la bibliographie p. 85; Pierre Maraval, Lieux saints et pèlerinages d'Orient. Histoire et géographie des origines à la conquête arabe, Paris, 1985, p. 281, avec la bibliographie n. 228-230.

5. Giorgi Čubinašvili, Bolnisskij Sion (Le Sion de Bolnisi), Tbilissi, 1940, p. 103-104; Giorgi Čubinašvili, C'romi, Moscou, 1969, p. 58.

6. Kiti Mačabeli, K'art'uli k'vajvarebi (Les croix en pierre géorgiennes), Tbilissi, 1998, p. 40.

7. Comme par exemple la croix syrienne du $\mathrm{IV}^{\mathrm{e}}$ siècle: Wolfgang Fritz Volbach, Avori di scuola ravennate nel V e VI secolo, Ravenne, 1977, cat. 7a; Age of Spirituality. Late Antique and Early Christian Art. Third to Seventh Century. Catalogue of the Exhibition at the Metropolitan Museum of Art, éd. Kurt Weitzmann, New York, 1979, n. 540; P. Maraval, Lieux saints, p. 281. 
L'importance de ce décor tient à son image figurative unique, qui nous permet de reconstituer sa fonction. Le personnage figuré sous l'arche peut être identifié à un donateur devant le monument qu'il a lui-même commandité; la scène dans son ensemble peut être interprétée comme une illustration du rituel de la vénération de la croix, elle-même représentée sur la droite. Il est difficile de reconstituer les gestes des fidèles devant ces monuments. Cependant, l'attitude de ce personnage peut être interprétée comme un acte de vénération; elle semble indiquer aussi, tout comme sa position sous une arche, le haut rang du donateur: les nobles sont représentés assis de cette manière et les rois sont souvent placés sous des arcs ou dans des niches dans l'iconographie sassanide et chrétienne. Le parallèle le plus proche est celui d'un ivoire copte du $\mathrm{VII}^{\mathrm{e}}$ siècle au Musée de Baltimore; selon l'avis général, il s'agit d'une copie de l'image triomphale de la fameuse coupe en argent ( $\mathrm{VI}^{\mathrm{e}} \mathrm{s}$.) du roi Chosroès $I^{8}$; d'autres objets, comme le plat sassanide du Musée de l'Ermitage $\left(\mathrm{VI}^{\mathrm{e}} \mathrm{s}\right.$.) ou encore la coupe de Chosroès II (?) à Téhéran $\left(\mathrm{VI}^{\mathrm{e}} \mathrm{s}\right.$.), offrent une iconographie similaire ${ }^{9}$. L'inscription en asomtavruli ${ }^{10}$ "Christ, prends pitié», confirme l'usage votif de la scène. Ainsi, cette représentation insolite reconstitue non seulement un modèle typologique de la stèle mais elle fournit de plus un témoignage explicite de la tradition locale ancienne du rituel de la vénération de la croix; elle illustre aussi la fonction cultuelle de ces monuments dans la Géorgie «féodale».

Une autre image (fig. 2), présente sur la stèle de Samc'evrisi $\left(\mathrm{V}^{\mathrm{e}}-\mathrm{VI}^{\mathrm{e}} \mathrm{s}\right.$.) confirme la tradition de la vénération de la croix en Géorgie paléochrétienne: le relief montre un médaillon avec une croix, érigée sur un poteau et vénérée par une femme qui tient une fleur dans sa main. Les vêtements portés par ce personnage, qui a probablement commandité le monument, représentent une variante du costume officiel byzantin, ce qui atteste l'influence des modèles byzantins tout en indiquant la haute position sociale de la donatrice.

8. Roman Ghirshman, Iran: Parthes and Sassanides, Paris, 1962, fig. 401402.

9. Splendeur des Sassanides. L'empire perse entre Rome et la Chine [224 642], 12 février au 25 avril 1993, Musées royaux d'art et d'histoire, Bruxelles, 1993, p. 206-207, cat. 61 ; R. Ghirshman, Iran, fig. 245-246.

10. L'asomt'avruli (signifiant «majuscules») est le plus vieil alphabet géorgien connu. 
L'installation des stèles en plein air, loin des églises et sur les hauteurs, sur les collines ou les montagnes les plus visibles ${ }^{11}$, conforte l'hypothèse de leur fonction cultuelle ${ }^{12}$. Les images figuratives de caractère dévotionnel et l'espace autour d'elles prouvent qu'elles étaient destinées à la prière. Elles marquaient un espace saint, où l'on pratiquait des rituels religieux.

Différents usages caractérisent ces objets. Compte tenu des programmes iconographiques diversifiés, ainsi que des nombreuses inscriptions qu'ils portent, ces objets semblent avoir servi à l'accomplissement de certains rituels, notamment baptismaux et funéraires, ou bien avoir eu une fonction votive et apotropaïque.

Les inscriptions, contemporaines des monuments qu'ils accompagnent, offrent de précieux témoignages pour définir leur fonction, et notamment leurs finalités: ils étaient utilisés pour prier, pour supplier, pour commémorer ${ }^{13}$. L'objet même est désigné comme une «croix du Christ ou croix toute-puissante», ce qui met en valeur sa force et son importance ${ }^{14}$. Les inscriptions contribuent ainsi à la définition de l'usage de l'objet. Le texte d'une prière à un saint ou à la croix place les monuments dans des contextes d'utilisation différents - commémoratif, funéraire, apotropaïque.

\section{LES SCÈNES DE BAPTÊME: LE RITE ET SON IMAGE}

Parmi ces rituels, l'un des plus importants était certainement le baptême des adultes organisé dans une rivière, suivant l'ancienne pratique $^{15}$. Le matériel archéologique confirme le lien entre les stèles et la cérémonie du baptême. Il est significatif que la plupart des stèles géorgiennes ornées d'une croix, dont la localisation

11. Niko Čubinašvili, Handisi, Tbilissi, 1972, p. 12.

12. II s'agit de stèles paléochrétiennes. Au Moyen Âge, elles sont contemporaines des églises, mais différentes d'un point de vue artistique: toujours couronnées d'une croix, elles ne portent pas de décor en relief (stèles de Kumurdo, Buzavet'i, Orja, Javaxet'i ( $\mathrm{x}^{\mathrm{e}}-\mathrm{XI}^{\mathrm{e}} \mathrm{s}$. $)$. Elles avaient probablement une autre fonction.

13. Nodar Šošiašvili, K'art'uli lapidaruli c'arc'erebis korpusi (Corpus des inscriptions lapidaires), Tbilissi, 1980, vol. I, p. 22-24.

14. L'accent mis sur la Croix commémore la Croix de bois plantée par sainte Nino et renvoie à celle du Golgotha.

15. Nina Iamanidzé, Les installations liturgiques sculptées dans les églises de Géorgie (VI ${ }^{e}$-XIII siècles), («Bibliothèque de l'Antiquité Tardive», 15), Turnhout, 2010, p. 27-33. 
est connue, soient toujours installées au bord des fleuves ${ }^{16}$. Les images du baptême représentées sur ces objets fournissent ainsi un témoignage visuel pour l'identification du rite. Sur les stèles de Berijvari ( $\mathrm{VI}^{\mathrm{e}}-\mathrm{VII}^{\mathrm{e}} \mathrm{s}$.) et d'Usanet'i (VIII ${ }^{\mathrm{e}} \mathrm{s}$.) (fig. 3), saint JeanBaptiste baptise le Christ jeune dans une petite cuve carrée, ornée au centre de grandes croix, alors que la scène évangélique se déroule dans le Jourdain. Il apparaît clairement ici que l'écart entre l'iconographie de la stèle et le texte évangélique ne peut être imputé qu'à l'usage rituel de cette dernière ${ }^{17}$.

Il apparaît ainsi que les images de baptême sur les stèles paléochrétiennes jouent un rôle de paradigme pour le rite baptismal géorgien qu'elles représentent. Elles révèlent que, dès le $\mathrm{vI}^{\mathrm{e}}$ siècle, le baptême des enfants dans des petites cuves était répandu au point que cet usage a inspiré l'iconographie du Baptême du Christ.

Les chercheurs ont unanimement conclu que les petites cuves n'ont servi qu'au baptême des nouveau-nés ${ }^{18}$. La réduction des dimensions des cuves à l'époque paléochrétienne est liée à l'évolution du rite baptismal, le baptême des adultes étant devenu une exception ${ }^{19}$. Nos recherches ont également confirmé que des cuves analogues étaient répandues en Géorgie, comme dans les autres régions de l'Orient chrétien ${ }^{20}$.

L'image du baptême représentée sur une cuve paléochrétienne en pierre de Žalet'i, fournit un autre témoignage visuel de ce rite. Cet objet remarquable, qui peut être identifié, sans difficultés, comme une cuve baptismale, est daté des $\mathrm{VI}^{\mathrm{e}}-\mathrm{VII}^{\mathrm{e}}$ siècles. Il offre un rare exemple d'interprétation du Baptême du Christ associé à une théophanie, et révèle l'originalité du traitement par lequel l'artiste aborde ce thème. L'iconographie se rattache aux modèles syro-

16. N. Čubinašvili, Handisi, p. 10.

17. Nina Iamanidzé, «Rite et aménagements baptismaux à l'époque paléochrétienne: le témoignage des sources archéologiques géorgiennes», Zograf, 32, 2008, p. 18.

18. Bruno Dufaÿ, Les baptistères paléochrétiens du Diocèse d'Orient. Étude archéologique et liturgique, Paris, 1977 (thèse dactylographiée), p. 75; JeanCharles Picard, Évêques, saints et cités en Italie et en Gaule : études d'archéologie et d'histoire, Rome, 1998, p. 160-162.

19. J.-Ch. Picard, Évêques, saints et cités, p. 160-162; Jacquelline LafontaineDosogne, «La tradition byzantine des baptistères et de leur décor, et les fonts de Saint-Barthélemy à Liège », Cahiers Archéologiques, 37, 1989, p. 48.

20. En Syrie, Palestine et Jordanie, voir: N. Iamanidzé, «Rite et aménagements baptismaux », p. 13-22; Iamanidzé, Les installations, p. 35-36. 
palestiniens: on y voit le Christ adulte dans le Jourdain, le SaintEsprit, sous la forme d'une colombe placée dans un cadre carré, descendu au-dessus de sa tête. Jean-Baptiste est à sa gauche, tandis que deux anges sur sa droite tendent les mains vers lui (fig. 4). L'image du Baptême offre ici un commentaire visuel du rite, elle indique la fonction liturgique sur l'objet lui-même. Le parallèle entre l'iconographie de l'objet et sa fonction y est explicite. La représentation joue le rôle d'un modèle typologique à suivre pour les fidèles. De ce fait, on perçoit immédiatement la nature de l'objet et l'image permet d'explorer les possibles interprétations de la liturgie qui accompagne le baptême.

\section{IMAGES SCULPTÉES, MIROIR DU RITUEL}

Un autre objet, dont la fonction originelle demeure inconnue, a été découvert dans une église d'Iq'alt'o où il a été remployé dans la partie supérieure de l'autel (fig. 5) ${ }^{21}$. Le programme iconographique de cette plaque du début du $\mathrm{x}^{\mathrm{e}}$ siècle constitue un exemple privilégié pour l'analyse des rapports entre les images et l'usage de l'objet, permettant de mettre en évidence les liens qui peuvent exister entre la sculpture et l'usage rituel de la plaque.

L'artiste d'Iq'alt'o, en composant ce programme, a principalement choisi des scènes du cycle christologique: l'enfance du Christ est représentée par les épisodes de l'Annonce aux bergers, par les mages venus d'Orient et par la Nativité. La Passion présente quelques particularités: on commence par l'Entrée à Jérusalem, avant de montrer le Chemin de Croix, la Crucifixion, puis les saintes femmes au tombeau. La vie publique du Christ se réduit quant à elle à la seule scène du miracle de la veuve de Naïn. Le décor de la plaque, comme la plupart des œuvres de cette époque, exprime donc la gloire du Christ en soulignant particulièrement l'idée du sacrifice, en mettant l'accent sur les thèmes de l'Incarnation et de la Rédemption. Cependant, le fait que la veuve soit mise en valeur, à la fois par son emplacement privilégié et par sa désignation par une inscription présente une originalité. La scène du miracle de la veuve de Naïn, rarement représentée, est l'une des scènes du cycle 
de la Résurrection et même la référence directe à la résurrection des morts. L'emploi de cette scène dans l'iconographie funéraire comme image-référence du Salut correspond à une tradition très ancienne. L'incorporation de cet épisode, assez insolite, dans le programme iconographique peut indiquer la fonction de l'objet. On peut supposer qu'une veuve ait été la donatrice de cet objet au nom des défunts, peut-être de membres de sa famille, parmi lesquels se trouverait son fils. Ce type de donation pour la mémoire de personnes décédées, souvent confirmé par les inscriptions dédicatoires qui accompagnent les images, peut être considéré comme habituel. Le choix de cette image donne en effet des raisons de penser que l'objet d'Iq'alt'o pouvait avoir une fonction funéraire. Le choix des inscriptions fournit davantage de précisions. La plupart de celles trouvées sur la plaque sont explicatives - elles désignent les personnages, sujets et objets représentés. Trois d'entre elles sont particulièrement remarquables: dans la scène de la veuve, au-dessus de la tête du Christ, on lit: «Christ, prends pitié de Kirile». Au coin inférieur du bras gauche de la croix du Christ, dans la Crucifixion, on lit: «Jésus Christ, prends pitié de Mik'aél». Sur le chanfrein entre les deux registres, sous les scènes de l'Annonce aux bergers et de la Nativité, on voit une longue inscription avec des lettres assez grandes qui disent: «Christ, procure le repos aux âmes d'Ivané, d'Iované, d'Ort'a, nommé Mika'él».

La dernière inscription citée est une prière à Dieu pour l'âme des trois défunts. Les noms de deux d'entre eux, Mik'el et Iované, sont mentionnés dans les deux autres inscriptions. La formule se rapproche de celles insérées sur les sarcophages et les stèles funéraires ${ }^{22}$.

22. Pour les stèles, voir: Klaus Wessel, L'art antique de la Basse-époque en Égypte, Bruxelles, 1963, p. 93-117, fig. 70-81; Ägypten Schätze aus dem Wüstensand: Kunst und Kultur der Christen am Nil. Katalog zur Ausstellung herausgegeben vom Gustav-Lübcke-Museum der Stadt Hamm und dem Museum für Spätantike und Byzantinische Kunst, Staatliche Museum zu BerlinPreußischer Kulturbesitz, Wiesbaden, 1996, p. 114-120; Nezih Firatli, La sculpture byzantine figurée au musée archéologique d'Istanbul. (Catalogue revu et présenté par Catherine Metzger, Annie Pralong et Jean.-Pierre Sodini), Paris, 1990, p. 93-94, cat. 171-177, pl. 54-55. Pour les sarcophages: Joseph Wilpert, I Sarcofagi Cristiani Antichi, Vatican, 1929-1936, vol. I, pl. 16 (1-2), pl. 17 (1) et pl. 136 (2); Giuseppe Bovini, Sarcofagi paleocristiani di Ravenna. Tentativo di classificazione cronologica, Rome («Amici delle Catacombe»), 1954, I, II et III. Aucun sarcophage n'a été retrouvé en Géorgie médiévale. 
Ces textes, ainsi que l'iconographie complexe de l'objet, permettent de supposer que cette plaque était la partie supérieure de la table de la chapelle annexe qui se trouvait, peut-être, dans le diakonikon de l'église. Elle aurait servi au dépôt des offrandes avant le service liturgique, plus probablement pour célébrer les offices commémoratifs. Ainsi, bien que l'image sur l'objet n'offre pas de commentaire visuel du rite, elle indique sa fonction cultuelle.

Le programme iconographique très complexe de l'une des deux plaques de $\mathrm{C}^{\prime}$ ebelda ( $\mathrm{IX}^{\mathrm{e}} \mathrm{s}$. ?) a été interprété de différentes manières $^{23}$ (fig. 6). Ce programme est composé principalement de scènes de l'Ancien et du Nouveau Testament. Cependant, sur la partie inférieure de la plaque apparaissent de curieux objets: on identifie ici la patène avec le pain, symbolisant, dans l'optique chrétienne, la communion par le pain ainsi que le vase qui renvoie à la communion par le vin. Deux personnages à gauche, donateurs ou ecclésiastiques, lèvent les mains en un geste de prière, l'un avec une croix au-dessus de sa tête, l'autre avec deux cierges dans les mains. On reconnaît dans ces objets des accessoires liturgiques, utilisés dans l'entrée des offrandes, en Géorgie et ailleurs; ces personnages sont, à notre avis, représentés pendant la célébration liturgique. L'image fait donc allusion au rite accompli dans le sanctuaire. On pense ici à un mort auquel on offrirait le service funéraire et pour lequel l'image voisine de la théophanie serait l'évocation de la lumière divine. Ce mort serait de la famille des fondateurs de l'église ${ }^{24}$.

23. La datation demeure très controversée, oscillant entre le $\mathrm{VI}^{\mathrm{e}}$ et le $\mathrm{XII}^{\mathrm{e}}$ siècle. Voir: Praskov'â. Uvarova, "Hristianskie pamâtniki. Abhaziâ. Adžariâ» (Les monuments chrétiens. Abxazie, Ač'ara), Materialy po Arheologii Kavkaza, 4, 1894, p. 22, fig. VII-VIII ; Leonid Šervašidzé, «Reznye kamni na holme Arasarakhu, bliz Cebel'dy» (Les pierres sculptées sur la colline Arasarakhu, près de C'ebelda), Izvestiâ Abhazskogo Instituta âzyka, literatury i istorii, 4, 1975, p. 90-105; Dmirtij Ajnalov, «Élinističeskie osnovy vizantijskogo iskusstva», Zapiski Kavkazskogo otdela russkogo geografičeskogo obščestva, 12, 3/4, Saint Petersbourg, 1901, p. 33-242; Ludmila Hruškova, Skul'ptura srednevekovoj Abhazii, Tbilissi, 1980, p. 43-85; Nicole Thierry, "Le culte du cerf en Anatolie et la Vision de saint Eustathe», Monuments et Mémoires 72, 1991, p. 80-84; Ludmila Khroushkova, Les monuments chrétiens de la côte orientale de la mer Noire. Abkhazie IV-XIV siècles, Turnhout («Bibliothèque de l'Antiquité Tardive», 9), 2006, p. 145-153. En l'état actuel, nous pencherons plutôt pour le $\mathrm{IX}^{\mathrm{e}}$ siècle; il semble que c'est avec les exemples de cette époque que les plaques de C'ebelda manifestent le plus de similitudes, voir: N. Iamanidzé, Les installations, p. 115-128.

24. Iamanidzé, Les installations, p. 124-125. 
La plaque présente donc les objets qui la relient directement à l'espace liturgique qu'elle inclut, ce qui met aisément en évidence le rapport entre l'image et le rituel; au sein d'un programme biblique, cette représentation fait allusion au rituel qui se déroule dans le sanctuaire; elle offre un exemple explicite du rapport entre l'iconographie sur l'objet et sa fonction qu'elle permet de reconstituer. Ce langage explicite permet aussi de distinguer cette scène des autres images présentes sur la plaque qui relèvent d'une thématique plus générale et illustrent les fondements du dogme chrétien.

On retrouve le reflet de cette tradition un peu plus tard, sur le décor du templon de Sxieri ( $\mathrm{x}^{\mathrm{e}} \mathrm{s}$.) (fig. 7-8). Seuls une plaque et un pilier ont subsisté de ce templon, composé à l'origine de deux panneaux et de quatre piliers ${ }^{25}$. Les archanges, surtout Gabriel sonnant de la trompette, les trois laïcs du registre inférieur gauche, dont l'un est montré dans une attitude d'orant, ainsi qu'un saint diacre non identifié tenant un encensoir, soulignent davantage le caractère invocatoire de l'image. Ces trois laïcs sont probablement les membres de la famille du donateur qui a dédié le templon à saint Georges, le patron de l'église. Compte tenu du contexte symbolique du programme iconographique de la plaque, on peut aussi supposer que ces personnages décédés attendaient leur jugement devant les archanges et ainsi lier le programme iconographique à l'évocation de l'âme du défunt et au Jugement Dernier. La croix avec le soleil au centre, qui divise le panneau en quatre carrés, apparaît quant à elle comme le symbole du Christ crucifié qui, comme le nouveau soleil, éclairera le monde et brûlera les pécheurs. Mais c'est l'association des quatre évangélistes avec les livres sur deux piliers et celle des saints avec les cierges et les rhipidia à la main sur les deux autres, qui permet de percevoir le lien direct avec le rituel. Ces objets du culte chrétien, dont le caractère est évident pour le ripidion qu'un officiant, habituellement le diacre, agite à la manière d'un éventail au cours de la célébration de la Grande Entrée, tout en se tenant devant l'autel en compagnie des offrandes, créent un ensemble cohérent qui peut être interprété comme une allusion à

25. René Šmerling, Malye formy v arhitekture srednevekovoj Gruzii (Formes mineures dans l'architecture géorgienne du Moyen Âge), Tbilissi, 1962, p. 76-82; Nina Iamanidze, «Icons of Ritual: the Programs of Earliest Georgian templa of Stone», Millennium, 8, 2010, p. 343-366. 
la célébration liturgique. La référence est celle de la liturgie de la Grande Entrée ou de l'Entrée de l'Évangile, qui est identifiée avec la Dernière Venue du Christ et le Jugement Dernier par Germanos et Maxime le Confesseur ${ }^{26}$. Ainsi, à Sxieri, l'image est non seulement liée à la fonction liturgique de l'espace auquel elle appartient, mais elle offre aussi un commentaire visuel du rite qui se déroule dans le sanctuaire.

Toujours dans le même contexte liturgique, l'église de saint Georges du village de Joisubani dans la région de Rač'a, à l'ouest de la Géorgie, recèle un exemple datant des $\mathrm{IX}^{\mathrm{e}}-\mathrm{X}^{\mathrm{e}}$ siècles, montrant l'une des rares représentations anciennes du Jugement Dernier ${ }^{27}$. L'épisode du Jugement proprement dit apparaît en une version extrêmement abrégée sur la partie supérieure de la fenêtre de la façade principale, à l'est (fig. 9). La plaque qui couronne la fenêtre montre l'image du Christ-Juge, désigné comme le Sauveur par une inscription en asomtavruli à droite de sa tête, soulignant de ce fait son rôle de Messie et de Rédempteur ${ }^{28}$. De tout le collège apostolique, seuls deux apôtres - Pierre et Paul, symboles de l'église apostolique et intercesseurs auprès de Dieu - ont été choisis pour l'accompagner. Le sens eschatologique de l'image est encore accentué par une autre inscription, à droite de Pierre, qui annonce le sujet: «le Jugement». Au-dessous de ce panneau, une autre plaque présente le thème de la pesée des actions; elle montre l'archange Michel, la balance à la main, face à ceux qui doivent subir cette épreuve. Le panneau de gauche montre l'archange Gabriel soufflant dans une trompette, annonçant l'apparition du Christ et le Jugement. Devant lui, un donateur se tient debout, un modèle de l'église dans les deux mains tendues, accompagné par l'inscription suivante: «Sainte église, prends pitié de Gabriel mamasaxlisi». Ce titre de la noblesse locale d'un commanditaire non identifié, que l'on peut traduire comme

26. Germanus, On the Divine Liturgy, trad. Paul Meyendorff, New York, Crestwood, 1984, p. 62.

27. Giorgi Boč'oridzé, «Rač' is istoriulu dzeglebi» (Les monuments historiques de Rač'a), Sak'art'velos muzeumis moambe, 8, 1935, p. 326-328; R. Šmerling, Malye formy, p. 80-81 (les auteurs mentionnent les reliefs à titre comparatif); Nat'ela Aladašvili, «Liturgiis asaxva», p. 77. Voir aussi Nina Iamanidzé, «Le thème du Jugement Dernier dans l'art religieux de Géorgie: témoignages archéologiques les plus anciens», sous presse dans Les Cahiers archéologiques, 57, 2015.

28. La paléographie des inscriptions indique le début du $\mathrm{x}^{\mathrm{e}}$ siècle, voir: N. Šošiašvili, Lapidaruli I, p. 43-48. 
«supérieur ecclésiastique», indique que dans le thème du Jugement Dernier se trouve intégrée l'image concrète d'un personnage historique, son rang social clairement défini, pour qu'il puisse à la fois être identifié et conserver sa hiérarchie. L'inscription s'adresse à l'église pour le Salut de son âme. L'objet, la maquette d'église, est tendu par le donateur qui espère miséricorde, vers Dieu, comme une offrande par laquelle se construit le lien à la divinité. Quant à l'archange Gabriel, il figure ici à la fois comme intercesseur et précurseur du Jugement. Les saints cavaliers, Georges, qui fait triompher la figure humaine, et Théodore tuant le dragon avec sa longue lance, figurent en bas de cette image. Quant à l'intégration des formules extrêmement abrégées de la délivrance miraculeuse des prophètes Daniel et de la tribulation de Jonas dans le programme de Joisubani, image qui est aujourd'hui perdue, elle met en valeur la symbolique de la Résurrection et en conséquence le lien direct au thème du Jugement ${ }^{29}$. La composition du Jugement Dernier de Joisubani incarne donc une interprétation relativement originale d'un thème où le concepteur, tout en connaissant les textes, ne les suit probablement pas directement ${ }^{30}$. Les intermédiaires sont à chercher dans une réunion de thèmes empruntés à différentes sources textuelles, mais aussi dans la liturgie.

Deux scènes sont réunies sur la plaque de la façade sud: l'Annonciation et la scène de la Rencontre (fig. 10). Ces deux images sont fondées sur la réciprocité thématique entre la Première

29. La présence du prophète Daniel montre aussi l'importance accordée à la Parousie, évoquée dans sa vision eschatologique.

30. La question de l'influence des sermons sur la Parousie d'Ephrem le Syrien sur l'iconographie des Jugements Derniers byzantins semble problématique à cause de l'absence de certains thèmes, notamment ceux de la pesée des actions, absente également de l'Apocalypse (Marcello Angheben, «Les Jugements derniers byzantins des $\mathrm{X}^{\mathrm{e}}$-XII ${ }^{\mathrm{e}}$ siècles et l'iconographie du Jugement immédiat», Cahiers Archéologiques, 50, 2002, p. 105-134, p. 128. Pour Éphrem le Syrien voir: Zaga Gavrilović, «St. Ephraim the Syrian's Thought and Imagery as an Inspiration to Byzantine Artists », Studies in Byzantine and Serbian Medieval Art, XVI, Londres, 2001, p. 244-270, p. 244-270), et bien présents à Joisubani comme d'ailleurs en Cappadoce, à İçeridere ou à Ylanlı kilise, par exemple (Catherine Jolivet-Lévy, «Nouvelles données sur le $\mathrm{IX}^{\mathrm{e}}$ siècle en Cappadoce: l'église d'İçeridere», Zbornik Radova Visantološkog Instituta, XLIV, 2007, p. 51, fig. 7-8 et «Premières images $\mathrm{du}$ Jugement Dernier en Cappadoce Byzantine ( $\mathrm{x}^{\mathrm{e}}$ siècle) $)$, Le Jugement Dernier entre Orient et Occident, éd. Valentino Pace, Paris, 2007, p. 47-52. En dehors de l'Évangile (Mathieu 25, 31-46; Marc 13, 24-37; Jean 5, 25-30), de l'Apocalypse et de la prophétie de Daniel, le thème du Jugement Dernier se fonde sur une série de sources textuelles. 
et la Seconde Venues: la Vierge, condition du Salut, intermédiaire par excellence entre les mondes céleste et terrestre grâce à l'Incarnation, implore le pardon pour les péchés des hommes; puis viennent Marie et Élisabeth, qui ont été les témoins de l'Incarnation. Nous constatons ainsi une association des thèmes de l'Incarnation et du Jugement.

La scène de la façade nord de l'église présente une procession de cinq personnages nimbés dans des tenues ecclésiastiques, les pieds dirigés vers la droite, avec des objets dans les mains qui renvoient clairement à la fonction liturgique de ce décor: celui du milieu tient un rhipidion, le premier à gauche tient un cierge, suivi par un personnage tenant un encensoir - attribut du diacre (fig. 11). Les deux derniers, à droite de la plaque, portent des objets ronds devant leur poitrine, qui sont particulièrement mis en valeur. Ils représentent le pain sacramentel, un des éléments essentiels destinés à la célébration eucharistique que l'officiant consacre pendant la cérémonie pour le partager avec les fidèles au cours de la communion, avant de le présenter à l'autel. Ces pains eucharistiques, connus et largement employés en Orient chrétien, sont habituellement marqués d'un sceau liturgique particulier appelé marqueur eucharistique et contenant un décor ornemental et parfois même figuratif. Nombre d'objets de ce genre en provenance de Jérusalem, d'Égypte copte et du Sinaï ont été découverts; certains sont conservés dans la collection de la Bibliothèque Nationale de France (Sn. 2), où l'on trouve un exemple presque identique à celui de Joisubani par ses dimensions et son ornementation (fig. 12) ${ }^{31}$. Ces pains d'eulogie font donc directement allusion à l'Eucharistie et renvoient explicitement au sacrement liturgique. L'une des trois inscriptions qui accompagnent ces personnages est une invocation: des deux côtés de la tête du deuxième personnage de droite on lit à gauche «sainte église»; à droite «prends pitié des maçons qui ont sculpté ce monument». De toute évidence, le décor sur la façade nord présente les serviteurs de l'église, probablement des moines,

31. L'objet est inédit et il n'est pas daté. Dimensions: diam. $137 \mathrm{~cm}$; haut. $60 \mathrm{~cm}$. Je remercie Béatrice Caseau de m'avoir communiqué les informations concernant les marqueurs eucharistiques extraites de son ouvrage en cours de préparation, intitulé: Identité et protection à Rome et à Byzance: les marqueurs du Cabinet des médailles. Deux autres articles consacrés à ces objets liturgiques seront publiés par l'auteur dans Liber Annus et Adalya. 
qui figurent avec les objets liturgiques pour célébrer le sacrement ecclésiastique. Cette procession liturgique pourrait faire allusion à la célébration de la Grande Entrée, identifiée au Jugement Dernier ${ }^{32}$.

Les objets inclus dans le répertoire de l'église permettent ainsi de mieux saisir le sens symbolique du décor de Joisubani, qui peut se résumer de la manière suivante: les artistes ont représenté la prière d'intercession au moment de la Parousie, en accentuant particulièrement le dogme de l'Incarnation et du Sacrifice du Christ, actualisé par le rite de l'Eucharistie. Cela se manifeste aussi dans la distribution des panneaux sur les façades où le concepteur semble bien avoir prévu la fonction liturgique des images, en plaçant l'épisode de la Rencontre avant l'Annonciation que les fidèles voyaient en premier en faisant le tour de l'église au moment de la célébration. La procession liturgique, présente sur un autre panneau, les guidait vers l'image principale du Jugement, où le Christ accueillait les fidèles, figurés sur le mur du sanctuaire. Ces images préparaient ainsi les fidèles à la célébration.

Le matériel archéologique géorgien propose un usage des images où la richesse et la complexité des programmes va au-delà de la simple définition iconographique. Les concepteurs introduisent des éléments renvoyant clairement au rituel; le recours fréquent aux représentations d'objets rituels révèle que le répertoire des monuments géorgiens au début du Moyen Âge puise non seulement aux sources des textes bibliques et hagiographiques mais aussi dans le rituel, notamment dans la divine liturgie. Cette référence à la liturgie permet d'établir la fonction de l'objet et, dans certains cas, d'émettre des hypothèses sur la reconstitution du rituel.

nina.iamanidz@college-de-france.fr

32. Selon l'interprétation de la Grande Entrée par Germanos et Maxime le Confesseur, dans la liturgie byzantine, la lecture de l'Évangile, le décent d'évêque du trône, l'expulsion du châtiment et des catéchumènes, symbolisent la Second Venue du Christ et du Jugement Dernier (ch. 14-15). 


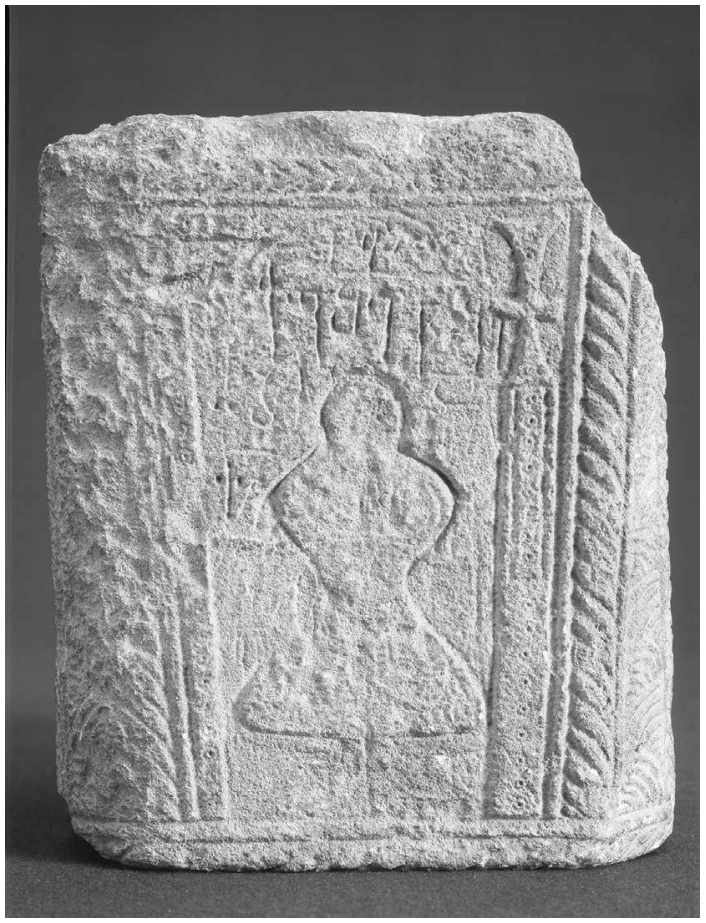

Fig. 1. Stèle de Dmanisi (région de K'vemo K'art'li), cliché N. Iamanidzé.

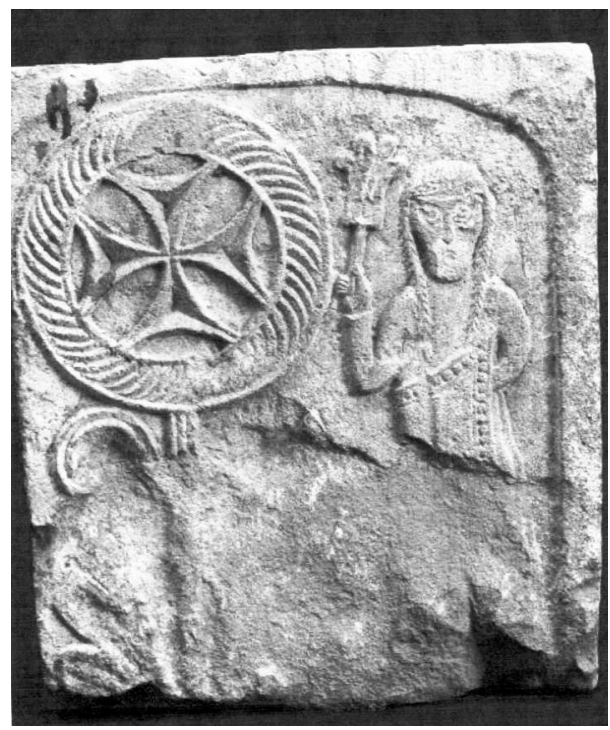

Fig. 2. Stèle de Samc'evrisi (région de Šida K'art'li), cliché N. Iamanidzé. 


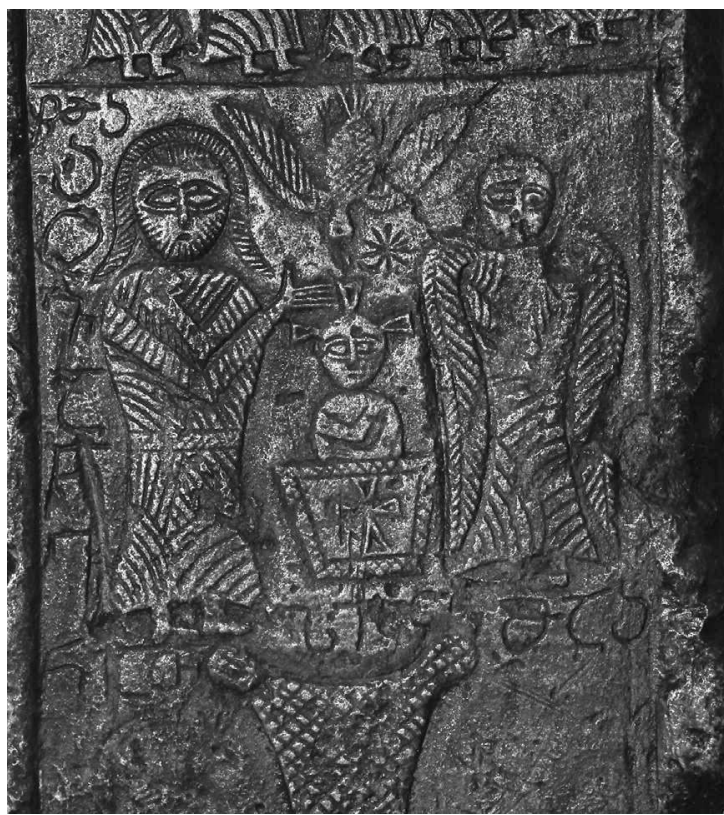

Fig. 3. Stèle de montagne, Usanet'i (région de Šida K'art'li), cliché N. Iamanidzé.

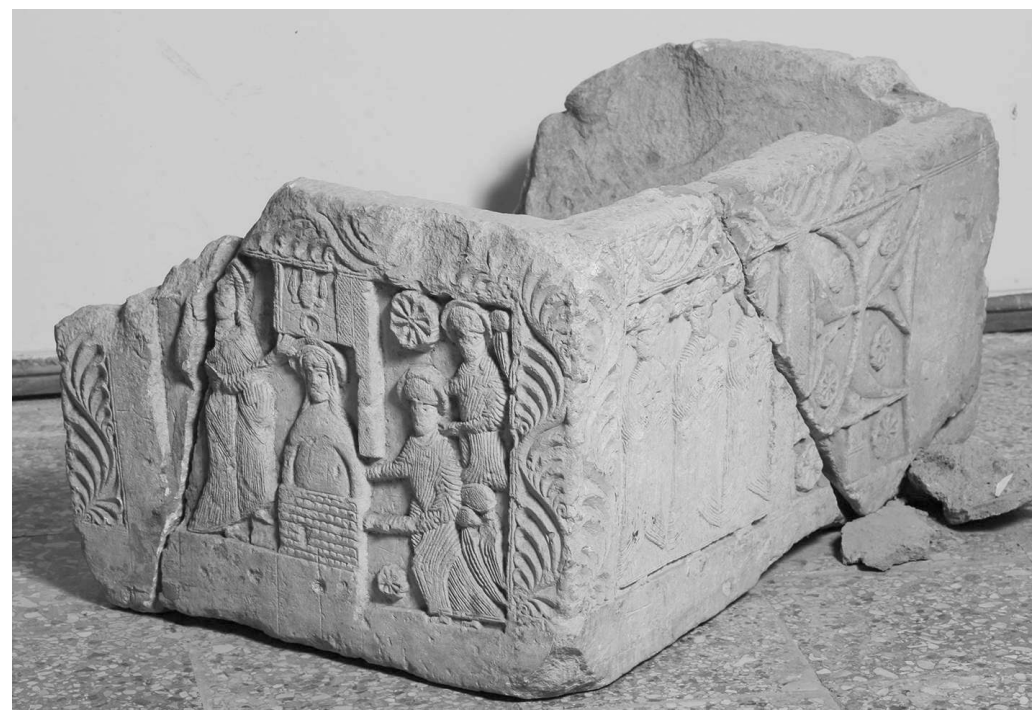

Fig. 4. Cuve de l'église de Žalet'i (région de T'ianét'i), cliché N. Iamanidzé. 


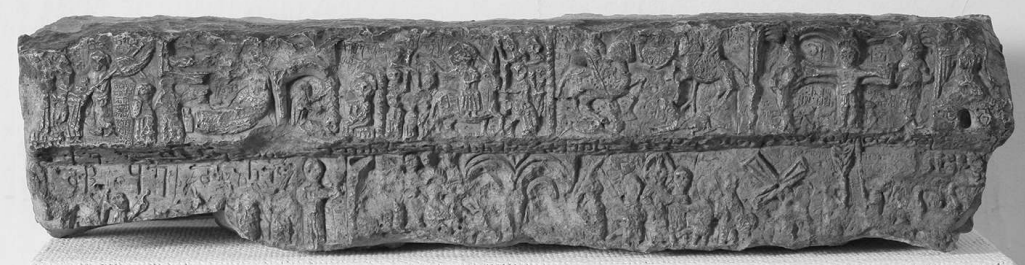

Fig. 5. Plaque d'Iq'alt'o (région de Kexét’i), cliché N. Iamanidzé.

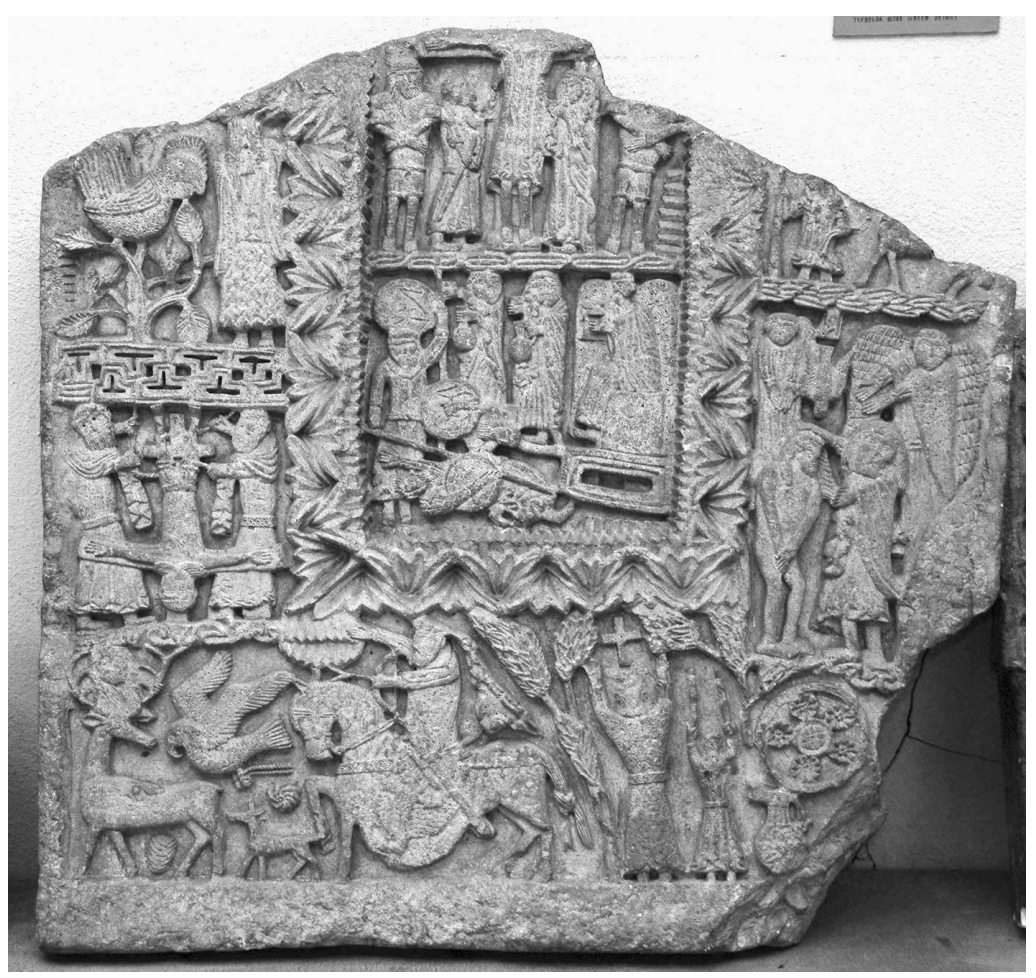

Fig. 6. Templon de C'ebelda (région d'Abxazie), cliché N. Iamanidzé. 

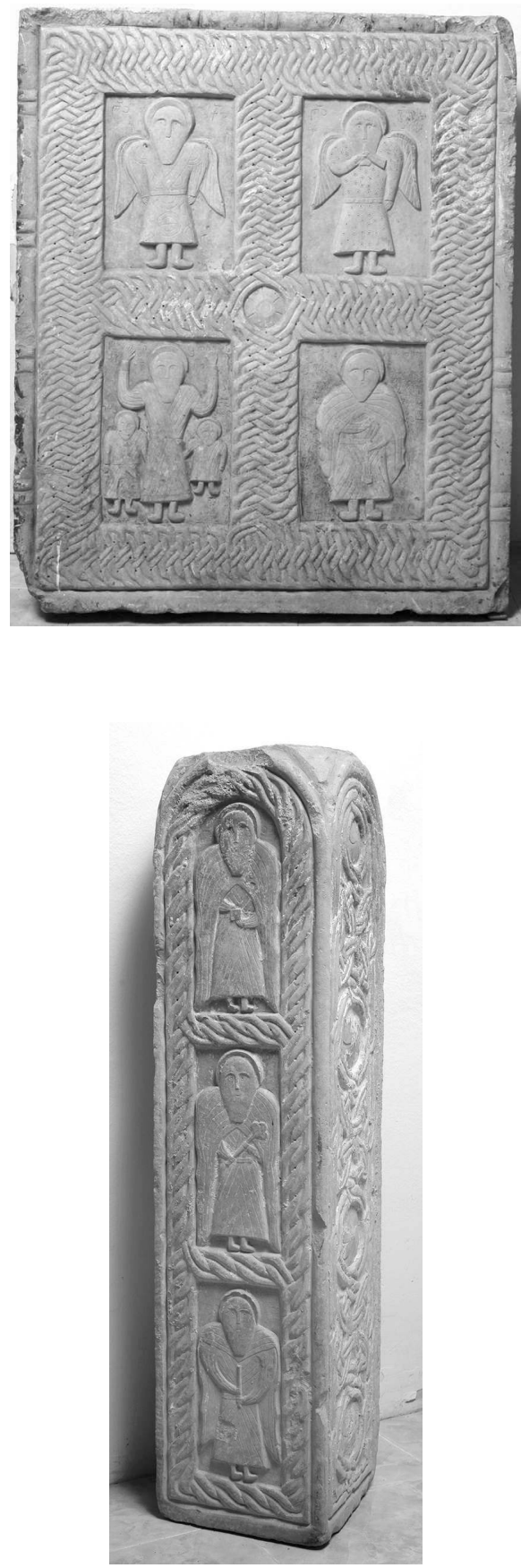

Fig. 7-8. Templon de Sxieri (région de Rač’a), clichés N. Iamanidzé. 


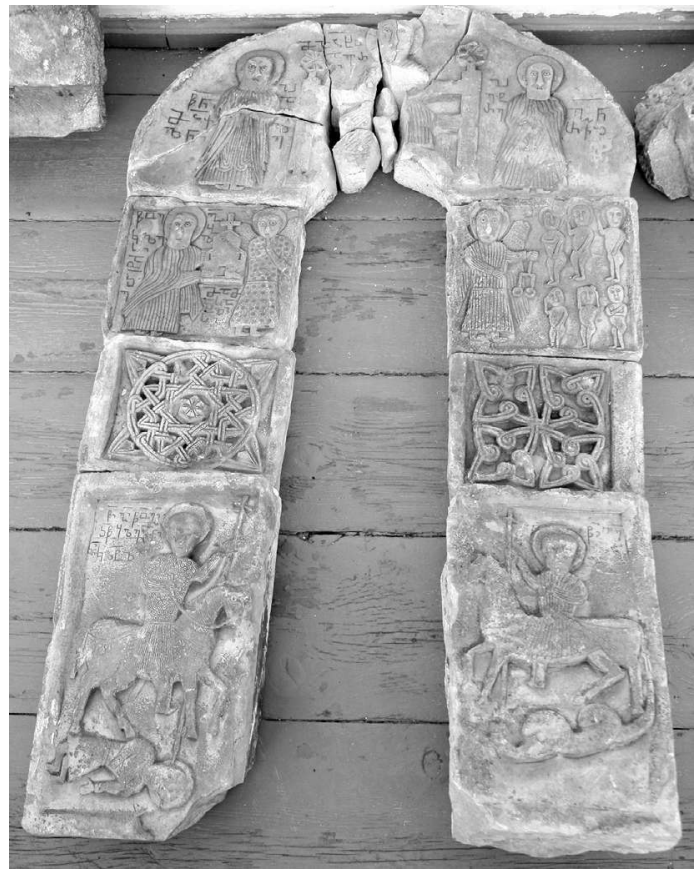

Fig. 9. Église de Joisubani (région de Rač’a). Relief de la façade est, cliché N. Iamanidzé.

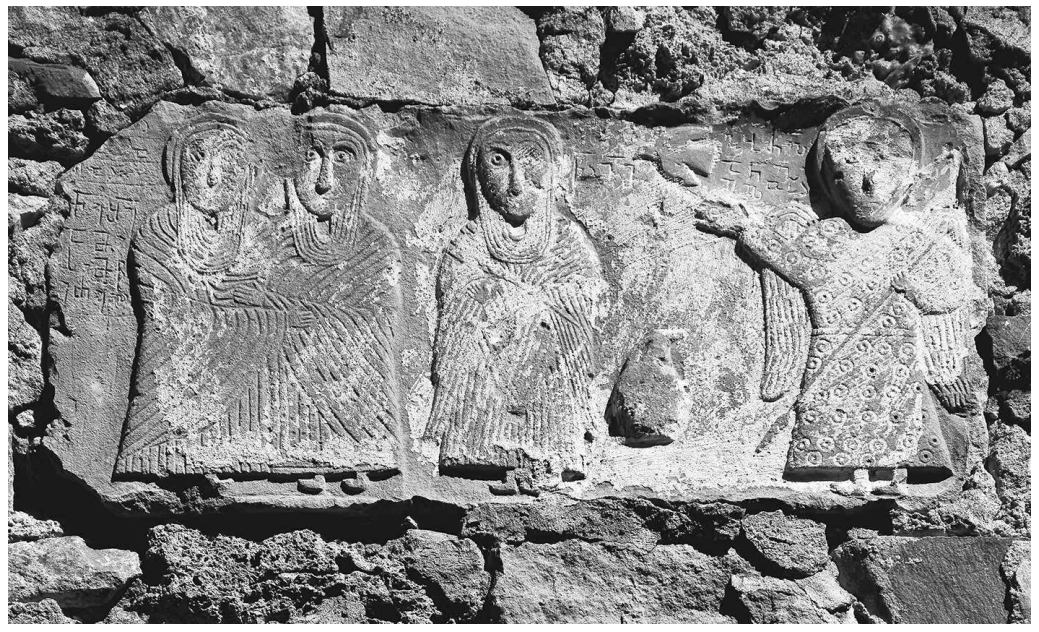

Fig. 10. Église de Joisubani (région de Rač'a). Façade sud, cliché N. Iamanidzé. 


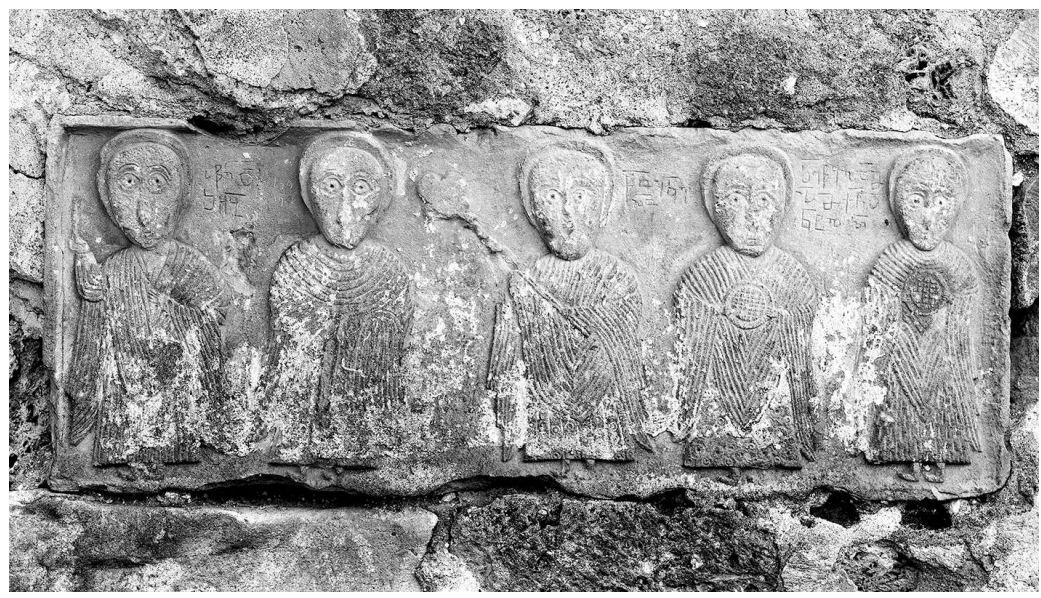

Fig. 11. Église de Joisubani (région de Rač’a). Façade nord, cliché N. Iamanidzé.

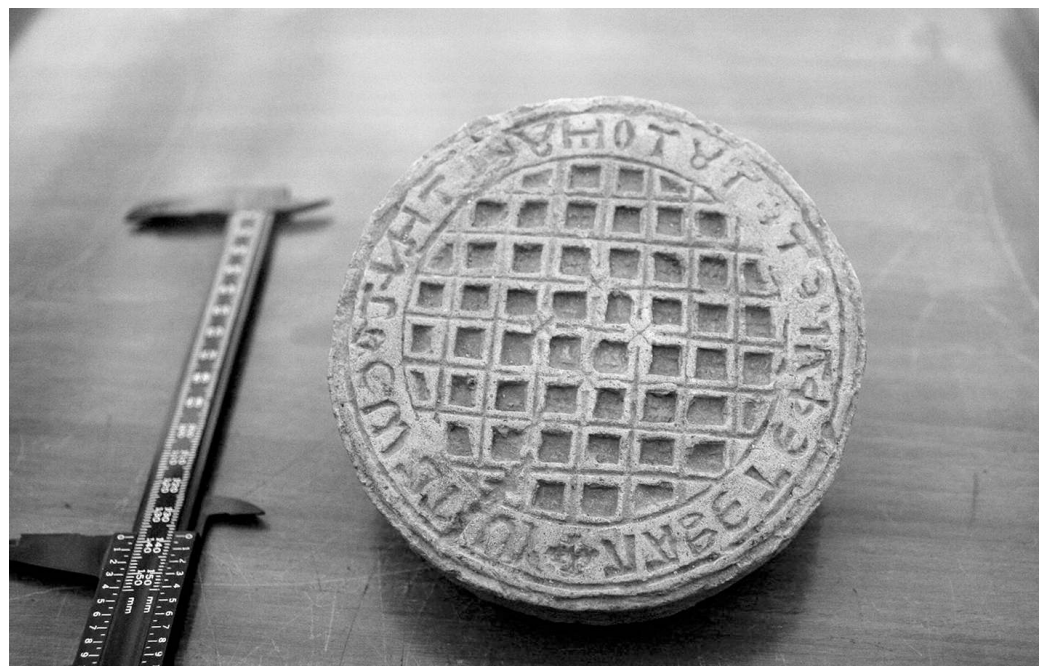

Fig. 12. Marqueur eucharistique, (C) Bibliothèque nationale de France (Cabinet des médailles), cliché Béatrice Caseau. 